\title{
Some Bipartite States Do Not Arise from Channels
}

\author{
Mary Beth Ruskai* \\ Department of Mathematics, Tufts University \\ Medford, Massachusetts 02155 USA \\ marybeth.ruskai@tufts.edu
}

November 6, 2018

Dedicated to Charles H. Bennett on his 60th Birthday

\begin{abstract}
It is well-known that the action of a quantum channel on a state can be represented, using an auxiliary space, as the partial trace of an associated bipartite state. Recently, it was observed that for the bipartite state associated with the optimal average input of the channel, the entanglement of formation is simply the entropy of the reduced density matrix minus the Holevo capacity. It is natural to ask if every bipartite state can be associated with some channel in this way. We show that the answer is negative.
\end{abstract}

PACS number 03.67; MSC classification 82P68.

\section{Background}

Recently, Matsumoto, Shimono and Winter (MSW) 4] pointed out an important connection between the channel capacity and of entanglement of formation, which allows one to draw some conclusion about the additivity of the latter from that of the former. There has also been speculation that the additivity of channel capacity and of entanglement of formation, are equivalent. A connection between additivity of entanglement of formation and multiplicativity of the p-norm measure of purity has also been given by Audenaert and Braunstein [1].

${ }^{*}$ Work partially supported by the U.S. National Science Foundation under Grant number DMS-03-14228. 
In view of this it is natural to ask if every bipartite state $\rho_{A B}$ can be associated with a channel in the sense of MSW. In particular is its reduced density matrix, $\rho_{A}$, the optimal average output of a channel whose capacity is related to the entanglement of formation of $\rho_{A B}$ as described in 4]? We will give a more precise formulation of this statement and show that the answer is negative.

Recall that a state is represented by a density matrix $\rho$, i.e., a positive semidefinite operator with $\operatorname{Tr} \rho=1$. The von Neumann entropy of $\rho$ is $S(\rho)=$ - $\operatorname{Tr} \rho \log \rho$. By an ensemble $\mathcal{E}=\left\{\pi_{i}, \rho_{i}\right\}$ we means a set of density matrices, $\rho_{i}$ and associated probabilities $\pi_{i}$. By a channel $\Phi$ we mean a completely positive, trace-preserving (CPT) map. The Holevo capacity of the map $\Phi$ is

$$
C_{\text {Holv }}(\Phi)=\sup _{\mathcal{E}}\left\{S[\Phi(\rho)]-\sum_{i} \pi_{i} S\left[\Phi\left(\rho_{i}\right)\right]\right\}
$$

where the supremum is taken over all ensembles $\mathcal{E}=\left\{\pi_{i}, \rho_{i}\right\}$ and $\rho=\sum_{i} \pi_{i} \rho_{i}$. The optimal average input is the state $\rho_{\mathrm{opt}}=\sum_{i} \pi_{i} \rho_{i}$ associated with the ensemble which attains the supremum in (10). The optimal average output is then $\Phi\left(\rho_{\text {opt }}\right)$.

The key to the MSW construction is the following result of Stinespring [7, which was subsequently used by Lindblad [3] in his work on relative entropy.

Theorem 1 Given a CPT map $\Phi$ on $\mathcal{B}(\mathcal{H})$, one can find an auxiliary space $\mathcal{H}_{B}$, a density matrix $\nu_{B}$ and a unitary map $U_{A B}$ on $\mathcal{H}_{A} \otimes \mathcal{H}_{B}$ such that

$$
\Phi(\rho)=\operatorname{Tr}_{B}\left[U_{A B}^{\dagger} \rho \otimes \nu_{B} U_{A B}\right]
$$

where $\operatorname{Tr}_{B}$ denotes the partial trace and we have identified $\mathcal{H}$ with $\mathcal{H}_{A}$.

Although we are interested in the case in which $\rho$ is a density matrix, the representation (2) is valid for all operators in $\mathcal{B}(\mathcal{H})$. When $\Phi(\rho)=\sum_{k} A_{k}^{\dagger} \rho A_{k}$, it is easy to construct a representation of the form (2), as discussed in section III.D of [6]. In fact, $U_{A B}^{\dagger} \rho \otimes \nu_{B} U_{A B}$ is the block matrix which has the form $\sum_{j k} A_{j}^{\dagger} \rho A_{k} \otimes|j\rangle\langle k|$.

Given a bipartite state $\gamma_{A B}$ on $\mathcal{H}_{A} \otimes \mathcal{H}_{B}$, its entanglement of formation satisfies

$$
\operatorname{EoF}\left(\gamma_{A B}\right)=\inf \left\{\sum_{k} \pi_{k} S\left(\operatorname{Tr}_{B} \gamma_{k}\right):\left\{\pi_{k} \gamma_{k}\right\} \text { ensemble with } \gamma_{A B}=\sum_{k} \pi_{k} \gamma_{k}\right\}
$$

Although it is customary to define the EoF using ensembles for which all $\gamma_{k}$ are pure states, there is no loss of generality in allowing arbitrary states.

Theorem 2 (Matsumoto, Shimono and Winter) Let $\Phi$ be a CPT map with a representation $\mathcal{H}_{B}, \nu_{B}, U_{A B}$ as in Theorem 1 . The Holevo capacity of $\Phi$ satisfies

$$
C_{\text {Holv }}(\Phi)=\sup \left\{S\left(\operatorname{Tr}_{B} \gamma_{A B}\right)-\operatorname{EoF}\left(\gamma_{A B}\right): \gamma_{A B}=U_{A B}^{\dagger} \rho \otimes \nu_{B} U_{A B}\right\}
$$

where $\rho$ is a density matrix on $\mathcal{H}=\mathcal{H}_{A}$. Moreover, the state $\widetilde{\gamma}_{A B}$ which attains this supremum satisfies $\operatorname{Tr}_{B} \widetilde{\gamma}_{A B}=\Phi\left(\rho_{\mathrm{opt}}\right)$ where $\rho_{\mathrm{opt}}$ is the optimal input of $\Phi$. 
It follows immediately that for the state $\widetilde{\gamma}_{A B}$

$$
\operatorname{EoF}\left(\widetilde{\gamma}_{A B}\right)=S\left[\Phi\left(\rho_{\text {opt }}\right)\right]-C_{\text {Holv }}(\Phi)
$$

It is thus natural to ask the following

Question 3 Given a bipartite state $\gamma_{A B}$ on $\mathcal{H}_{A} \otimes \mathcal{H}_{B}$, is there a CPT map $\Phi$ on $\mathcal{B}\left(\mathcal{H}_{A}\right)$ such that $\operatorname{Tr}_{B} \gamma_{A B}$ is the optimal average output state of $\Phi$ and

$$
\operatorname{EoF}\left(\gamma_{A B}\right)=S\left(\operatorname{Tr}_{B} \gamma_{A B}\right)-C_{\text {Holv }}(\Phi) ?
$$

We will show that the answer to this question is negative.

Our counter-example is based on a result in [5] and [9] which comes from the fact that when $\rho=\sum_{k} \pi_{k} \rho_{k}$

$$
S(\rho)-\sum_{k} \pi_{k} S\left(\rho_{k}\right)=\sum_{k} \pi_{k} H\left(\rho_{k}, \rho\right)
$$

where the relative entropy is defined as

$$
H(\omega, \rho)=\operatorname{Tr} \omega[\log \omega-\log \rho] .
$$

Theorem 4 Let $\Phi$ be a CPT map with optimal average input $\rho_{\mathrm{opt}}$. Then

$$
C_{\text {Holv }}(\Phi)=\sup _{\omega} H\left[\Phi(\omega), \Phi\left(\rho_{\text {opt }}\right)\right]
$$

It follows as an immediate corollary, that if $\mathcal{E}=\left\{\pi_{k}, \rho_{k}\right\}$ is any optimal ensemble for the channel $\Phi$, then $H\left[\Phi\left(\rho_{k}\right), \Phi\left(\rho_{\text {opt }}\right)\right]=C_{\text {Holv }}(\Phi)$ is independent of $k$, i.e., the optimal average output is "equi-distant" in the sense of relative entropy from all outputs $\Phi\left(\rho_{k}\right)$ in the ensemble.

\section{Counter-example}

An affirmative answer to Question 3 above would imply that if the ensemble $\left\{\pi_{k},\left|\psi_{k}\right\rangle\left\langle\psi_{k}\right|\right\}$ is optimal for $\operatorname{EoF}\left(\gamma_{A B}\right)$, then $\left\{\pi_{i}, \operatorname{Tr}_{B}\left|\psi_{k}\right\rangle\left\langle\psi_{k}\right|\right\}$ would be an optimal output ensemble for the corresponding map $\Phi$. It would then follow from the equi-distance corollary to Theorem 8 that

$$
H\left(\omega_{k}, \gamma_{A}\right)=-S\left(\omega_{k}\right)-\operatorname{Tr}_{A} \omega_{k} \log \gamma_{A}=C .
$$

where $\omega_{k}=\operatorname{Tr}_{B}\left|\psi_{k}\right\rangle\left\langle\psi_{k}\right|$ and $C$ is a constant which is independent of $k$. (In fact, $C$ is the Holevo capacity of $\Phi$ if such a channel exists.) We will present an example of a bipartite qubit state which does not satisfy (10). 
It may be that counter-examples to (10) can already be found in the literature on entanglement. However, we will take advantage of a result of Wootters [10 to construct a rather simple qubit counter-example which does not require extensive numerical computation. In [10], Wootters obtained an explicit formula for the EoF of a bipartite qubit state using a quantity called the concurrence. Moreover, he showed that the EoF could be achieved using an ensemble of at most four pure states $\left|\psi_{k}\right\rangle\left\langle\psi_{k}\right|$, for which $S\left(\operatorname{Tr}_{B}\left|\psi_{k}\right\rangle\left\langle\psi_{k}\right|\right)=\operatorname{EoF}\left(\gamma_{A B}\right)$. Thus, for such an ensemble, (10) holds if and only if

$$
\operatorname{Tr}_{A}\left[\left(\operatorname{Tr}_{B}\left|\psi_{k}\right\rangle\left\langle\psi_{k}\right|\right) \log \gamma_{A}\right]
$$

is independent of $k$. We can assume without loss of generality that $\gamma_{A}$ is diagonal with eigenvalues $\frac{1}{2}(1 \pm x)$. Then $\operatorname{Tr} \omega \log \gamma_{A}$ depends only on the diagonal elements of $\omega$ which can be written as $\frac{1}{2}(1 \pm d)$. In fact,

$$
\begin{aligned}
\operatorname{Tr} \omega \log \gamma_{A} & =\frac{1+d}{2} \log \frac{1+x}{2}+\frac{1-d}{2} \log \frac{1-x}{2} \\
& =\log 2-\frac{1}{2}\left[\log \left(1-x^{2}\right)+d \log \frac{1+x}{1-x}\right]
\end{aligned}
$$

which depends linearly on $d$ for fixed $x \neq 0$. Thus, when $\gamma_{A} \neq \frac{1}{2} I$, (11) is independent of $k$ if and only if all $\omega_{k}=\operatorname{Tr}_{B}\left|\psi_{k}\right\rangle\left\langle\psi_{k}\right|$ have the same diagonal elements. However, we also know that $S\left(\omega_{k}\right)=\operatorname{EoF}\left(\gamma_{A B}\right)$ is independent of $k$, which implies that all $\omega_{k}$ have the same eigenvalues. Thus, all the reduced density matrices $\operatorname{Tr}_{B}\left|\psi_{k}\right\rangle\left\langle\psi_{k}\right|$ must have the form $\frac{1}{2}\left(\begin{array}{cc}1+d & e^{i \theta_{k}} t \\ e^{-i \theta_{k}} t & 1-d\end{array}\right)$ for some fixed $t$. It is not hard to find an example for which this does not hold.

Let $\left|\beta_{0}\right\rangle=\frac{1}{\sqrt{2}}(|00\rangle+|11\rangle)$ and $\left|\beta_{3}\right\rangle=\left(\sigma_{z} \otimes I\right)\left|\beta_{0}\right\rangle=\frac{1}{\sqrt{2}}(|00\rangle-|11\rangle)$ be the indicated maximally entangled Bell states, and

$$
\gamma_{A B}=\frac{5}{8}\left|\beta_{0}\right\rangle\left\langle\beta_{0}\left|+\frac{1}{16}\right| \beta_{3}\right\rangle\left\langle\beta_{3}\left|+\frac{1}{4}\right| 01\right\rangle\left\langle 01\left|+\frac{1}{16}\right| 10\right\rangle\langle 10| .
$$

The reduced density matrix is $\gamma_{A}=\operatorname{Tr}_{B} \gamma=\frac{1}{2}\left(I+\frac{3}{16} \sigma_{z}\right) \neq \frac{1}{2} I$. One can show that the optimal EoF decomposition of (13) has the form $\gamma_{A B}=\sum_{k=1}^{4} \pi_{k}\left|\psi_{k}\right\rangle\left\langle\psi_{k}\right|$ with $\left|\psi_{1}\right\rangle=a_{0}\left|\beta_{0}\right\rangle+a_{3}\left|\beta_{3}\right\rangle$ so that $\omega_{1}=\operatorname{Tr}_{B}\left(\left|\psi_{1}\right\rangle\left\langle\psi_{1}\right|\right)$ is diagonal in the basis $|0\rangle,|1\rangle$ (i.e, $t=0$ in the matrix above). However, the remaining $\psi_{k}$ are superpositions which contain Bell states $\left|\beta_{k}\right\rangle$ and the product states $|01\rangle,|10\rangle$ in a form which necessarily yields a reduced density matrix $\omega_{k}$ which is not diagonal. By the discussion above, this implies a negative answer to Question [3.

To actually find the entanglement of formation and optimal decomposition of $\gamma_{A B}$, let

$$
\widetilde{\gamma}=\frac{5}{8}\left|\beta_{0}\right\rangle\left\langle\beta_{0}\left|+\frac{1}{16}\right| \beta_{3}\right\rangle\left\langle\beta_{3}\left|+\frac{1}{4}\right| 10\right\rangle\left\langle 10\left|+\frac{1}{16}\right| 01\right\rangle\langle 01|
$$


be the density matrix with all spins flipped. The concurrence $\mu$ can be expressed in terms of the eigenvalues of $(\sqrt{\gamma} \widetilde{\gamma} \sqrt{\gamma})^{1 / 2}$. Following Wootters [10], one finds $\mu=\frac{5}{8}-\frac{1}{16}-\frac{1}{8}-\frac{1}{8}=\frac{5}{16}$. Let

$$
h(x)=-\frac{1+x}{2} \log \frac{1+x}{2}-\frac{1-x}{2} \log \frac{1-x}{2} .
$$

Then, proceeding as described in [10], one finds

$$
\operatorname{EoF}\left(\gamma_{A B}\right)=h\left(\sqrt{1-\mu^{2}}\right)=h\left(\frac{\sqrt{231}}{16}\right) \approx 0.1689<0.9745 \approx h\left(\frac{3}{16}\right)=S\left(\gamma_{A}\right)
$$

The optimal ensemble has weights

$$
\pi_{1}=0.1527, \pi_{2}=\pi_{3}=\pi_{4}=0.2824
$$

associated with the projections for the following pure states

$$
\begin{aligned}
& \left|\psi_{1}\right\rangle=0.8101\left|\beta_{0}\right\rangle+0.5863\left|\beta_{3}\right\rangle \\
& \left|\psi_{2}\right\rangle=-0.7870\left|\beta_{0}\right\rangle+0.1087\left|\beta_{3}\right\rangle+0.5432|01\rangle+0.2716|10\rangle \\
& \left|\psi_{3}\right\rangle=0.7870\left|\beta_{0}\right\rangle-0.1087\left|\beta_{3}\right\rangle+0.5432 e^{i \pi / 3}|01\rangle+0.2716 e^{-i \pi / 3}|10\rangle \\
& \left|\psi_{4}\right\rangle=-0.7870\left|\beta_{0}\right\rangle+0.1087\left|\beta_{3}\right\rangle-0.5432 e^{-i \pi / 3}|01\rangle-0.2716 e^{i \pi / 3}|10\rangle
\end{aligned}
$$

One can easily verify that the diagonal elements of $\omega_{1}=\operatorname{Tr}_{B}\left|\psi_{1}\right\rangle\left\langle\psi_{1}\right|$ are not equal to those of $\omega_{k}=\operatorname{Tr}_{B}\left|\psi_{k}\right\rangle\left\langle\psi_{k}\right|$ for $k=2,3,4$. One can also compute the reduced density matrices $\omega_{k}$ and see that $\omega_{1}$ is diagonal in the basis $|0\rangle,|1\rangle$, but the others are not. Alternatively, one can observe that if a reduced density matrix is diagonal in the basis $|0\rangle,|1\rangle$, then any purification must have the form

$$
|\Psi\rangle=a|0\rangle \otimes\left|\phi_{1}\right\rangle+b|1\rangle \otimes\left|\phi_{2}\right\rangle
$$

with $\left|\phi_{1}\right\rangle,\left|\phi_{2}\right\rangle$ orthogonal. By rewriting

$$
\begin{aligned}
\left|\psi_{2}\right\rangle & =-0.4796|00\rangle-0.6333|11\rangle+.5432|01\rangle+.2716|10\rangle \\
& =-|0\rangle \otimes(0.4796|0\rangle-.5432|1\rangle)+|1\rangle \otimes(.2716|0\rangle-0.6333|1\rangle),
\end{aligned}
$$

one sees that $\psi_{2}$ does not have the form (16) with orthogonal $\left|\phi_{k}\right\rangle$. The actual reduced density matrices have the form $\omega_{1}=\frac{1}{2}\left[I+\frac{\sqrt{231}}{16} \sigma_{z}\right]=\left(\begin{array}{cc}0.9750 & 0 \\ 0 & 0.0250\end{array}\right)$, $\omega_{k}=\left(\begin{array}{cc}0.5251 & e^{i \theta_{k}} 0.4743 \\ e^{-i \theta_{k}} 0.4743 & 0.4749\end{array}\right)$ with $\theta_{2}=\pi, \theta_{3}=\frac{\pi}{3}, \theta_{4}=-\frac{\pi}{3}$. 


\section{Remarks on representations}

In the canonical method of constructing a representation of the form (2), the reference state $\nu_{B}$ is pure and $d_{B}$ the dimension of $\mathcal{H}_{B}$ is equal to the number of Kraus operators. Then the lifted state $\gamma_{A B}=U_{A B} \rho \otimes \nu_{B} U_{A B}^{\dagger}$ for which $\Phi(\rho)=\operatorname{Tr}_{B} \gamma_{A B}$ has rank at most $d=d_{A}$, the dimension of the original Hilbert space. This is clearly a very restricted class of bipartite states. Moreover, generically, $d_{B}>d$ since many maps require the maximum number $d^{2}$ of Kraus operators. Thus the canonical representation yields only bipartite states which are far more singular than typical states.

Some maps $\Phi(\rho)$ can be represented in the form (2) using a mixed reference state $\nu_{B}$. Indeed, given a mixed bipartite state $\nu_{B}$ and unitary operator $U_{A B}$, (2) can be used to define a channel $\Phi$. Unfortunately, relatively little is known about such mixed state representations. It has been suggested that one might be able to represent a channel $\Phi$ in the form (2) using a space of dimension $d_{B}=d$ if mixed states are used. However, this is known not to be true in general [8, 11].

Now suppose that a qubit channel $\Phi$ can be represented (possibly using a mixed reference state $\nu_{B}$ ) in the form (2) using an auxiliary qubit space $\mathcal{H}_{B}$ so that $d_{B}=2$. Then the argument given before (11) can be used to show that for the optimal input distribution $\left\{\pi_{k} \rho_{k}\right\}$

$$
\operatorname{Tr} \Phi\left(\rho_{k}\right) \log \Phi\left(\rho_{\mathrm{opt}}\right)
$$

must be independent of $k$. One can easily check that the 3 -state examples given in 2 do not have this property. Most non-unital qubit maps for which the translation of the image of the Bloch sphere lacks symmetry will also violate (17). This implies that such channels require an auxiliary space with $d_{B}>2$. One expects $d_{B}=4$, consistent with the fact that such maps also require 4 Kraus operators. This gives another, somewhat indirect, proof that some CPT maps require an auxiliary space with $d_{B}>d$.

It would be of some interest to characterize the maps which admit mixed state representations with $d_{B}=d$, as well as the bipartite states corresponding to the optimal average output.

Acknowledgment: It is a pleasure to thank Professor Christopher King for stimulating and helpful discussions. 


\section{References}

[1] K. M.R. Audenaert and S. L. Braunstein "On strong superadditivity of the entanglement of formation" quant-ph/0303045.

[2] C. King, M. Nathanson and M.B. Ruskai "Qubit channels can require more than two inputs to achieve capacity" Phys. Rev. Lett. 88, 057901 (2002).

[3] G. Lindblad, "Completely positive maps and entropy inequalities" Commun. Math. Phys. 40, 147-151 (1975).

[4] K. Matsumoto, T. Shimono and A. Winter, "Remarks on additivity of the Holevo channel capacity and of the entanglement of formation" quant$\mathrm{ph} / 020614$

[5] M. Ohya, D. Petz and N. Watanabe, "On capacities of quantum channels" Prob. Math. Stats. 17, 170-196 (1997).

[6] M.B. Ruskai, "Inequalities for quantum entropy: a review with conditions for equality" J. Math. Phys. 43, 4358-4375 (2002).

[7] W.F. Stinespring, "Positive functions on $C^{*}$-algebras" Proc. Amer. Math. Soc. 6, 211-216 (1955).

[8] B.M. Terhal, I.L. Chuang, D.P. DiVincenzo, M. Grassl, J.A. Smolin "Simulating quantum operations with mixed environments" Phys.Rev. A60, 881-884 (1999).

[9] B. Schumacher and M. D. Westmoreland, "Optimal signal ensembles" Phys. Rev. A 63, 022308 (2001).

[10] W. Wootters, "Entanglement of formation of an arbitrary state of two qubits" Phys. Rev. Lett. 80, 2245-2248 (1998).

[11] C. Zalka and E. Rieffel "Quantum operations that cannot be implemented using a small mixed environment" J. Math. Phys. 43, 4376-4381 (2002). 